\title{
Eurostudia
}

\section{Roman à clés et écriture du réel : La constellation du Lynx de Louis Hamelin et Forêt contraire d'Hélène Frédérick}

\section{Robert Dion}

Volume 13, numéro 1-2, 2018-2019

Cultures en contact, entre régulations et représentations

URI : https://id.erudit.org/iderudit/1067288ar

DOI : https://doi.org/10.7202/1067288ar

Aller au sommaire du numéro

\section{Éditeur(s)}

Le Centre canadien d'études allemandes et européennes

ISSN

1718-8946 (numérique)

Découvrir la revue

Citer cet article

Dion, R. (2018). Roman à clés et écriture du réel : La constellation du Lynx de Louis Hamelin et Forêt contraire d'Hélène Frédérick. Eurostudia, 13(1-2), 369-391. https://doi.org/10.7202/1067288ar
Résumé de l'article

Le roman à clés a mauvaise presse. Pourtant, il a innervé, tout au long du $\mathrm{XX}^{\mathrm{e}}$ siècle, des livres fondamentaux comme $A$ la Recherche du temps perdu de Marcel Proust ou certains romans de James Joyce et de Virginia Woolf. Plus largement, de nombreux romans contemporains ont recours à des dispositifs de " floutage " pour faire entrer le réel, et plus particulièrement les personnes réelles, dans la fiction. Ainsi l'enjeu, dans les deux romans québécois qui se voient analysés dans cet article, consiste à trouver une autre manière d'inscrire le réel dans la fiction. Les exemples de « réticences » et d'hésitations vis-à-vis du nom propre correspondent ici à des stratégies diverses d'appréhension de l'histoire ou du rapport intime entre les êtres, qui commandent certes des détours mais qui, en même temps, exigent une certaine transparence de la référence pour laisser percevoir un poids de réalité garant d'une authenticité dont les lecteurs d'aujourd'hui auraient, dit-on, une insatiable faim. Ces stratégies, on le verra, invitent à revoir les rapports entre le réel et la fiction, à remettre en cause les frontières censées les cloisonner.
Tous droits réservés $@$ Le Centre canadien d'études allemandes et européennes, 2019
Ce document est protégé par la loi sur le droit d'auteur. L'utilisation des services d’Érudit (y compris la reproduction) est assujettie à sa politique d'utilisation que vous pouvez consulter en ligne.

https://apropos.erudit.org/fr/usagers/politique-dutilisation/ 


\title{
Roman à clés et écriture du réel: La constellation du Lynx de Louis Hamelin et Forêt contraire d'Hélène Frédérick
}

\author{
Robert Dion \\ CRILCQ, Université du Québec à Montréal
}

\section{Résumé}

Le roman à clés a mauvaise presse. Pourtant, il a innervé, tout au long du $\mathrm{XX}$ siècle, des livres fondamentaux comme À la Recherche du temps perdu de Marcel Proust ou certains romans de James Joyce et de Virginia Woolf. Plus largement, de nombreux romans contemporains ont recours à des dispositifs de « floutage » pour faire entrer le réel, et plus particulièrement les personnes réelles, dans la fiction. Ainsi l'enjeu, dans les deux romans québécois qui se voient analysés dans cet article, consiste à trouver une autre manière d'inscrire le réel dans la fiction. Les exemples de « réticences » et d'hésitations vis-à-vis du nom propre correspondent ici à des stratégies diverses d'appréhension de l'histoire ou du rapport intime entre les êtres, qui commandent certes des détours mais qui, en même temps, exigent une certaine transparence de la référence pour laisser percevoir un poids de réalité garant d'une authenticité dont les lecteurs d'aujourd'hui auraient, dit-on, une insatiable faim. Ces stratégies, on le verra, invitent à revoir les rapports entre le réel et la fiction, à remettre en cause les frontières censées les cloisonner.

\section{Abstract}

The roman à clé receives a lot of negative press. Yet, throughout the twentieth century, it has innervated fundamental books such as Marcel Proust's À la recherche du temps perdu or 
some novels by James Joyce and Virginia Woolf. More widely, many contemporary novels use devices of "blurring" to bring reality, and more particularly real people, in the fiction. Thus the issue, in the two Quebec novels analyzed in this article, is to find another way to include reality in fiction. The examples of "reluctance" and hesitation vis-à-vis the proper name correspond here to various strategies of perception of history or of the intimate relationship between beings, which certainly require detours but which, at the same time, require a certain transparency of the reference to let perceive a weight of reality - the guarantee of an authenticity for which the readers of today would have, so one says, an insatiable hunger. These strategies, as we shall see, invite us to review the relationship between reality and fiction, to question the boundaries that are supposed to partition them.

L'écriture $^{1}$ de la personne réelle, c'est un fait généralement connu, passe par le caractère attesté du nom propre. C'est le cas dans l'écriture biographique et dans l'autofiction, notamment. Si un récit de vie paraît correspondre à la trajectoire d'une personne ayant véritablement existé mais que le nom propre est modifié, on parlera plutôt alors de roman biographique ou autobiographique et non de biographie, d'autobiographie ou d'autofiction². Mais qu'arrive-t-il lorsque le récit n'est pas centré sur un seul protagoniste, que son cadre est globalement romanesque, que la mention «roman » figure dans le paratexte et que, sous les noms fictifs des personnages, on reconnaît des personnes? Peut-on se contenter de discréditer ces œuvres comme des romans à clés ${ }^{3}$ et en abandonner la lecture aux seuls serruriers et amateurs de ragots ${ }^{4}$ ?

\footnotetext{
${ }^{1}$ Ce texte reprend, avec des modifications notables, un chapitre de mon dernier livre, Des fictions sans fiction ou le partage du réel (2018)

${ }^{2}$ L'autofiction présuppose l'homonymie entre l'auteur, le narrateur et le personnage; c'est une condition essentielle selon Serge Doubrovsky, l'inventeur et le premier théoricien du genre.

${ }^{3}$ C'est-à-dire comme des œuvres qui relèvent d'une pratique d'écriture où la correspondance entre le personnage fictif et la personne réelle est la fois suggérée par une certaine transparence référentielle et
} 
Ce sont les effets de tels dispositifs de «floutage» permettant aux personnes réelles d'envahir le roman qui me retiendront ici. Peut-on alors toujours parler d'écriture du réel, c'est-à-dire d'une écriture qui prétendrait rendre compte de la réalité, que ce soit celle de la biographie effective d'une figure existante, celle d'un épisode historique ou celle de l'actualité la plus brûlante? Je ne m'attacherai pas ici aux fictions basées sur des faits divers où le camouflage des noms répond d'abord à la nécessité de se soustraire à d'éventuelles poursuites. L'enjeu, dans les textes qui m'occupent, n'est pas tant d'enrayer la machine judiciaire que de trouver une autre manière d'inscrire le réel dans la fiction. Les exemples de "réticences » et d'hésitations vis-à-vis du nom propre sur lesquels j'entends me pencher correspondent à des stratégies diverses d'appréhension de l'histoire ou du rapport intime entre les êtres, qui commandent certes des détours mais qui, en même temps, exigent une certaine transparence de la référence pour laisser percevoir un poids de réalité garant d'une authenticité dont les lecteurs d'aujourd'hui auraient une insatiable faim ${ }^{5}$.

Je m'arrêterai à La constellation $d u$ Lynx de Louis Hamelin (2012 [2010]) $)^{6}$ et à Forêt contraire d'Hélène Frédérick (2015 [2014]) ${ }^{7}$, deux romans qui se caractérisent

celée par la dénomination, créant par là une hésitation entre vérité et fiction et une disparité d'effet pragmatique, certains lecteurs pouvant actionner la clé et d'autres non.

${ }^{4}$ Sur le roman à clés et les aléas de sa réception, voir le collectif publié sous la direction de Glinoer et Lacroix (2014).

${ }^{5}$ Je renvoie ici au titre fameux du livre de David Shields, Reality Hunger (2010).

${ }^{6}$ Dorénavant, les références à cet ouvrage seront notées par le sigle $C L$ et le folio, entre parenthèses dans le corps du texte. Je me permets de renvoyer à deux articles que j'ai consacrés à La constellation du Lynx (2015a, 2015b). Né en 1959, Louis Hamelin est un romancier et un chroniqueur important dont l'œuvre, amorcée en 1989 par un roman, La rage, qui a obtenu le prestigieux prix du Gouverneur général, a suscité l'attention soutenue des critiques et des universitaires. La constellation $d u$ Lynx a obtenu quatre prix littéraires, dont le prix Ringuet.

${ }^{7}$ Dorénavant, les références à cet ouvrage seront notées par le sigle $F C$ et le folio, entre parenthèses dans le corps du texte. Installée en France où elle publie aux Éditions Verticales (les versions en poche étant publiées au Québec par Héliotrope), Hélène Frédérick, née en 1976, a publié, avant Forêt contraire, un roman consacré à l'artiste et costumière allemande Hermine Moos (1888-1928) et au peintre Oskar Kokoschka, La poupée de Kokoschka (2014 [2010]). 
par un recours particulier à des figures réelles et aux noms propres qui les désignent (mais qui, bien entendu, ne se limitent pas à cela). Il importe de noter que ce ne sont pas les études d'onomastique qui constitueront ici le socle ni le nœud de l'analyse; je ne m'étendrai pas, ou si peu, sur la motivation du nom romanesque ni sur ses connotations, mais plutôt sur l'usage du nom et ses répercussions sur la lecture du texte comme témoignage indirect, récit à clés ou variation imaginative sur le réel.

\section{Hamelin : la fiction dans l'histoire}

La constellation $d u$ Lynx a connu un succès populaire considérable, de même qu'une réception critique et médiatique exceptionnelle ${ }^{8}$. Les uns l'ont lu comme le grand roman tant attendu sur Octobre 1970, comme une enquête et une somme rigoureuses faisant échec à la propagande des gouvernements de même qu'au récit héroïque des felquistes ${ }^{9}$, alors que les autres l'ont assimilé, dans un registre nettement moins laudatif, à un délire conspirationniste. Toutes divergentes qu'elles aient été, ces diverses lectures n’ont pu faire l'économie d'un questionnement sur les raisons pour lesquelles Hamelin, au terme des huit années de recherche préalables à l'écriture, a choisi d'écrire un roman plutôt

\footnotetext{
8 Parmi les textes littéraires qui abordent la crise d'Octobre 1970, on peut mentionner entre autres, de Pierre Turgeon, Un dernier blues pour Octobre (1990), de Louis Caron, le troisième tome de la saga des Fils de la liberté, Le coup de poing (1990), et d'Andrée Ferretti, "Octobre de lumière " (dans La vie partisane, 1990), trois œuvres publiées en 1990 pour commémorer les vingt ans de la crise. Mais il y a eu bien d'autres textes qui ont pris Octobre 1970 sinon pour sujet, du moins pour cadre de la fiction. Dans un article de 2002, Jacques Pelletier insiste sur le fait que, "considérée globalement tant dans les œuvres instituées, professionnelles, que dans les textes de circonstances, forcément plus artisanaux, cette production littéraire [sur la crise d'Octobre] s'avère décevante, sinon carencée. Elle n'apparaît pas à la hauteur des événements dont elle donne une représentation maladroite » (2007 [2002] : 179). C'est sans doute là ce qui explique le retentissement médiatique exceptionnel de l'entreprise d'Hamelin, qui est apparue comme la première vraie tentative, totalisante et engagée, de prendre à bras-le-corps l'événement dans toutes ses ramifications - événementielles aussi bien qu'idéologiques et symboliques. Voir aussi, toujours de Jacques Pelletier, « La crise d'Octobre 1970 et la littérature québécoise » (1995 [1982] : 141-162). ${ }_{9}^{9}$ C'est le nom que l'on donne aux membres du Front de libération du Québec, le FLQ, groupe terroriste actif dans les années 1960 et au début des années 1970.
} 
qu'un essai. L'écrivain s'en explique à Noémi Mercier dans l'entrevue qu'il lui a accordée pour le magazine L'Actualité :

- Si vous êtes convaincu de ce que vous avancez, pourquoi ne pas avoir écrit un essai historique plutôt qu'un roman?

- Je le ferai peut-être un jour. L'essai m'aurait obligé à être plus prudent, je l'avoue. Par le roman, j'ai pu m'aventurer dans des zones que les historiens officiels ne peuvent explorer, parce que leur travail repose essentiellement sur les archives écrites. Je n'ai pas, non plus, le fardeau de la preuve journalistique.

Mon imagination de romancier m'a servi d'instrument pour fouiller cette affaire. Seule cette forme littéraire me permettait d'ouvrir des pistes et d'embrasser l'histoire dans toutes ses contradictions, ses ambiguïtés, ses coins d'ombre (Hamelin et Mercier $2010: n . p$.).

Les motifs qu'allègue ici Hamelin ne sont guère originaux et on les trouve chez nombre d'écrivains qui prétendent, par la fiction, accéder à une vérité supérieure aux évidences factuelles de l'histoire. Plus inhabituelle est la fougue de l'auteur à affirmer, dans plusieurs de ses entrevues et dans les textes polémiques qui ont accompagné la publication de son roman, que sa fiction est plus exacte que les diverses publications, celles-là involontairement fictionnelles, qui, jusqu'ici, ont été produites par des témoins, des journalistes et des historiens qui auraient eu tout intérêt, selon lui, à donner des versions tronquées ou gauchies des événements.

Par ailleurs, l'essai qu'annonçait Hamelin dans cet entretien a effectivement été publié sous le titre Fabrications (2014), mais il ne s'agit pas tant d'un essai de type historiographique que d'une réflexion fragmentée "sur la fiction et l'histoire », comme l'énonce le sous-titre. L'auteur y revient sur l'apport possible de la fiction dans la résolution des impasses historiques ${ }^{10}$ et, sur un mode plus spécifique, sur le caractère ambivalent de sa posture de romancier. Dans un

${ }^{10}$ Voir par exemple Hamelin (2014 : 143s.). 
dialogue simulé entre lui et Sam Nihilo, son alter ego de La constellation du Lynx, à propos des nombreux entretiens accordés à la presse à la sortie du livre où était réaffirmé le caractère véridique des conclusions du roman, Hamelin fait dire à son personnage : «À quoi bon faire œuvre d'imagination si c'était pour renier la part de fiction à la première occasion?» (2014:56) Or, c'est une telle posture entre fiction et non-fiction qui permet justement à Hamelin de jouer sur tous les tableaux, de se réclamer à la fois de Norman Mailer, qui a combiné fiction documentée et écriture documentaire inspirée des techniques de la fiction ${ }^{11}$, et de Don DeLillo, qui, lui, s'ancre fermement sur le terrain de l'art autonome tout en affirmant les pouvoirs de la littérature d'imagination à décoder le réel ${ }^{12}$.

Bref, Hamelin affirme avec force la vérité, herméneutique et factuelle, d'un récit à teneur historique pourtant inscrit dans un cadre qui s'affiche d'emblée comme romanesque. L'on pourrait même préciser: comme hyperboliquement romanesque. En effet, le roman s'ouvre par une narration «impossible » signalée d'entrée de jeu : « Je m’appelle Marcel Duquet et je vais mourir dans environ cinq minutes » (CL:17). Cette mort annoncée survient ironiquement dans une localité nommée L'Avenir. Plusieurs éléments de ce début indiquent les partis pris qui seront ceux d'Hamelin :

1. la transmission du récit d'un mort en sursis signale la toutepuissance du romancier, sa capacité de débusquer les narrations empêchées ou occultées;

2. le changement de nom du personnage historique, le militant indépendantiste et sympathisant felquiste Michel Viger, produit ce léger flou qui permet d'aménager un espace de jeu propice à la saisie romanesque de l'histoire;

\footnotetext{
11 À la manière du New Journalism dont Mailer est, avec Tom Wolfe, l'un des plus éminents représentants.

${ }^{12}$ Hamelin songe en particulier à Libra, le roman de DeLillo sur l'assassinat de John F. Kennedy.
} 
3. le déplacement du lieu où eut lieu le décès de Viger, de SaintNazaire-de-Bagot à la localité voisine de L'Avenir, accentue l'ironie du destin d'un personnage justement sans avenir, décelant par là une intention purement romanesque et critique;

4. la conversion en assassinat de ce qui est en règle générale considéré comme un accident - à l'été 1975, Viger aurait été écrasé par son tracteur alors qu'il travaillait dans les champs de son frère - indexe d'ores et déjà l'herméneutique suspicieuse qui sera au principe de la recherche d'Hamelin.

La liste des personnages et la chronologie des principaux événements survenus entre le 5 et le 17 octobre 1970, qui occupent les premières pages du livre, si elles fournissent des repères utiles, se nient cependant en partie comme balises historiques puisqu'elles ne donnent pas les noms véritables des protagonistes de la crise. La chronologie évite certes d'utiliser les faux noms forgés par Hamelin, parlant par exemple de l'« Enlèvement du numéro deux du gouvernement québécois» plutôt que de Paul Lavoie (dans le roman) ou de Pierre Laporte (dans la réalité), mais elle mentionne tout de même John Travers au lieu de James Richard Cross ${ }^{13}$. Quant à la liste qui précède cette chronologie, elle énumère aussi bien les acteurs réels que les personnages de pure fiction ${ }^{14}$, non sans aplanir jusqu'à un certain point ces différences de statut en affublant de noms inventés les personnes réelles répertoriées. Elle rappelle d'ailleurs davantage le théâtre et ses mises en scène que le genre romanesque ou l'écrit historique.

\footnotetext{
13 « 5 octobre 1970 : Enlèvement du délégué commercial de Grande-Bretagne, John Travers, par le Front de libération du Québec » (CL: n. p.).

14 Ainsi, dans la catégorie «Les littéraires": «Chevalier Branlequeue, éditeur, poète, prof de littérature/Samuel Nihilo, tâcheron de la plume/Marie-Québec Brisebois, femme de théâtre/Frédéric Falardeau, chercheur » (CL: n. p.).
} 
Certaines de ces renominations sont transparentes : la Cellule Libération du Front de libération du Québec (FLQ) devient ainsi la Cellule Rébellion et la Cellule Chénier, la Cellule Chevalier; Jacques Lanctôt est baptisé Lancelot, Paul Rose, Jean-Paul Lafleur, et son frère Jacques, René Lafleur; le ministre assassiné Pierre Laporte, on l'a vu, se change en Paul Lavoie. D'autres transformations, en revanche, sont plus opaques: Richard Godefroid pour Francis Simard, Luc Goupil pour Richard Bros, entre autres. Si la fonction conférée à certains protagonistes de la liste aide au repérage («Maître Mario Brien, avocat des terroristes » ne peut être que maître Robert Lemieux, «Albert Vézina, premier ministre du Québec » renvoie à Robert Bourassa), d'autres appellations obligent à poursuivre l'enquête, parce qu'en plus d'être assez éloignées des noms originaux elles désignent des acteurs à peu près inconnus du public non spécialiste : c'est le cas, par exemple, de Francis Braffort, qui désigne François Mario Bachand, ou de Raymond Brossard alias Zadig et de Daniel Prince alias Madwar, qui cachent Michel Lambert alias Salem et Normand Roy alias Selim.

La première chose qui frappe dans cette onomastique, c'est son caractère hétérogène, non seulement sur l'axe transparence-opacité, mais aussi quant au mode de formation des noms inventés. Ainsi, de Pierre Laporte à Paul Lavoie, la distance est réduite; de Paul Rose à Jean-Paul Lafleur, il n'y a que l'ajout d'un prénom et le passage du particulier (« rose») au général («fleur»); de Lanctôt à Lancelot, la paronomase suffit à motiver la renomination, tandis que, de James Cross à John Travers, c'est la traduction Cross-Travers(e) qui garantit la transparence de l'identification. Par contre, on ne peut établir aucun lien immédiat entre, disons, Francis Simard et Richard Godefroid.

L'hétérogénéité de l'onomastique tient aussi, comme l'a remarqué Élisabeth Nardout-Lafarge en évoquant le modèle que constitue pour Hamelin Le ciel de Québec de Jacques Ferron (1969), à son caractère ambigu « alternant vrais et faux 
noms, transparents ou grotesques, [ce qui] suspend le pacte de lecture entre le rétablissement des identités réelles et l'adhésion à des personnages fictifs dans une constante interrogation de la dimension romanesque de la réalité » (2013: 180). De plus, comme le livre d'Hamelin, le roman de Ferron est, pour une part au moins, un «roman à clés où l'on peut reconnaître des hommes de lettres (Saint-Denys Garneau, mais aussi Camille Roy, Gilles Marcotte et Claude Gauvreau), des artistes (Borduas et Paterson Ewen) et des politiciens » (NardoutLafarge 2013 : 180). En fait, il y a bien peu de personnages de La constellation $d u$ Lynx qui laissent libre cours à une totale immersion dans la fiction : même le personnage vaguement grotesque de Chevalier Branlequeue est inspiré de Jacques Ferron, de Gaston Miron et d'Hubert Aquin, alors que Sam Nihilo est, pour sa part, un avatar de l'auteur qui semble en souligner la vacuité (nihil). Il n'y a guère que Marie-Québec Brisebois et Fred Falardeau, Jacques «Coco » Cardinal et son ex-épouse qui, parmi les protagonistes majeurs du récit, semblent ne pas avoir de contrepartie dans le réel.

On l'a dit, nombre d'éléments du texte viennent atténuer la dimension réaliste de La constellation $d u \operatorname{Lynx}$, voire son caractère sérieux. Le personnage loufoque de Chevalier Branlequeue, homosexuel rentré, poète rare et conspirationniste patenté, ainsi que le cercle de ses étudiants obsédés par Octobre 1970 au point de se nommer les Octobierristes, contribuent à inscrire une distance ironique entre le récit et l'auteur, comme si ce dernier n'adhérait pas complètement à ce qui se veut pourtant une reconstitution totalisante d'une crise politique depuis ses signes avant-coureurs jusqu'au présent; ou comme si, malgré la somme de recherches consacrées à l'évocation de la crise, il ne la prenait pas au sérieux, y voyant au fond une révolution d'opérette. Des éléments fantastiques telles les apparitions de Paul Lavoie en spectre exigeant d'être vengé participent également de cette relative mise en échec du réalisme. Mais, en même temps, le roman ne cesse de multiplier les accroches au réel en ayant entre autres 
recours à des toponymes et à des anthroponymes avérés, en produisant des documents authentiques, en rappelant des événements attestés, en développant des analyses assez souvent plausibles de points d'histoire restés nébuleux et, surtout, en multipliant les clés de lecture qui ouvrent sur un arrière-plan dont on a de bonnes raisons de croire qu'il est fondé dans la réalité. Si donc, comme je l'ai mentionné, La constellation du Lynx invite d'entrée de jeu à une appréhension romanesque de l'histoire, elle n'en suscite pas moins, sous la forme d'un chiasme, une lecture historicisante de la fiction.

Ce recours ostensible à des clés est certes susceptible d'occasionner une lecture «référentielle» $d u$ texte à travers laquelle est d'abord visée une « véridiction » qui prétend confirmer ou infirmer l'énoncé (Lacroix 2014 : 14, note 16). Mais, tout bien considéré, La constellation $d u$ Lynx a plutôt appelé, dans les faits, une réception sélective, qui a soit exhaussé tout ce qui rattache le roman au réel, soit, à l'inverse, accentué ce qui l'en détournait. Dans ce dernier cas, le livre est apparu telle une captivante (et amusante) spéculation conspirationniste, évidemment paranoïaque, élaborée à partir d'un certain nombre de faits historiques peu ou prou transposés. Mais on peut imaginer une lecture qui, se refusant au « tout ou rien », enjamberait le fossé entre réalité et fiction, accepterait que certaines portes donnent sur la réalité et d'autres sur l'imaginaire, mettant ainsi en lumière «l'interdépendance de ces deux catégories » et traduisant « les romans en faits réels et l'histoire en fiction» (Latham 2014 [2009] : 34-35). Du coup, on sortirait de l'appréhension traditionnellement dépréciative du roman à clés pour y voir non pas un "genre» facile et racoleur, mais « un principe déconstructif de contamination et de renversement» des catégories génériques (Latham 2014 [2009] : 34) ${ }^{15}$. Apparaîtrait alors de manière plus distincte l'enjeu

\footnotetext{
${ }^{15}$ C'est la thèse de Sean Latham dans son ouvrage The Art of Scandal, où il montre comment Joyce dans Portrait de l'artiste en jeune homme et Ulysse, Woolf dans Orlando ou Proust dans La recherche usent du roman à clés dans une optique de renouvellement des formes. Voir la traduction de l'introduction de ce livre dans le collectif dirigé par Glinoer et Lacroix (Latham 2014 [2009]).
} 
proprement littéraire du roman d'Hamelin, à ne pas confondre avec les intentions explicites de l'auteur, qui consiste à montrer comment les fictions font partie de l'histoire, s'y amalgamant à un point tel que les éradiquer reviendrait à la mutiler, alors que leur multiplication contribuerait, au contraire, à constituer un certain « réalisme critique » de l'indétermination historique.

\section{Frédérick : décaler l'autre}

Dans Forêt contraire, Hélène Frédérick manifeste une même prédilection pour la mise en scène de personnes réelles et pour le jeu sur les noms propres. Dans ce roman, Frédérick installe un cadre nettement fictionnel qui, à la rigueur, pourrait passer pour autobiographique, la narratrice de ce récit autodiégétique partageant avec l'auteure quelques biographèmes : le même âge ou à peu près, le lieu de naissance, l'installation à Paris. Ces similitudes restent cependant vagues à dessein, le floutage de l'identité de la narratrice étant l'un des procédés les plus insistants de Forêt contraire. En effet, alors que manifestement la protagoniste a un vaste appétit pour les noms - elle prend plaisir à nommer les plantes de la forêt, les poissons d'un aquarium (FC:49), etc. - , elle se refuse obstinément à établir son identité, inaugurant sa narration par un «Je me présente: je n'ai plus de nom » $(F C: 9)$. Cette amnésie revendiquée (FC:9) est d'autant plus étonnante que le personnage vient de quitter Paris pour se réinstaller provisoirement dans un lieu relié à son histoire personnelle, à Inverness, au Québec: «difficile, poursuit-elle, d'oublier son nom quand on a défait sa valise dans l'ancien chalet des parents et du frère » (FC:9). Lors d'une rencontre avec André, un voisin comédien, elle se refuse également à révéler son nom, lui suggérant plutôt de lui en inventer un. Le comédien opte spontanément pour «Sophie » (FC:42) et c'est le nom qu'elle adoptera tout au long du récit. Cette renomination servira à consolider la dissociation de la narratrice entre « elle », la jeune femme au début de la vingtaine d'avant l'émigration à Paris, et «moi », la narratrice de 28 ans, 
voire entre les trois termes d'une «triade » constituée par « elle, Sophie et moi » $(F C: 118-119)^{16}$.

En soi, l'anonymat de l'instance narrative, en régime homo- ou autodiégétique, n'est ni étonnant ni même rare. Comme le remarque Frank Wagner, un tel anonymat instaure entre le narrateur et le lecteur une valeur d'intimité tout à fait bienvenue qu'une mention explicite de l'identité narratoriale risquerait de mettre en péril en nuisant à l'immersion fictionnelle par l'introduction $\mathrm{d}^{\prime}$ " une distance, [ $\left.\mathrm{d}^{\prime}\right]$ un hiatus entre la voix et ses destinataires » (2008: 21). Ce qui, en revanche, est plus inusité, c'est l'insistance sur cet anonymat, c'est la surjustification de cette absence de nom par la réitération de l'amnésie et le comblement de cette lacune au moyen de la renomination par un tiers, par l'attribution d'un nom d'emprunt que la narratrice, pâte en apparence aisément malléable, n’hésitera pas un instant à faire sien.

En face de cette instance narrative à l'identité fuyante, il y a donc André et, surtout, la figure d'un homme suicidé qu'elle a connu dans son autre vie montréalaise, avant son départ pour Paris, Lukas Bauer. Ce dernier revient hanter sa mémoire pourtant défaillante alors qu'elle retrouve, parmi les livres qu'elle a récupérés à Montréal et emportés à Inverness, l'autobiographie, jamais lue jusqu'alors, de cet intellectuel et écrivain allemand qui avait croisé son chemin à quelques reprises dans la métropole. Le lecteur un tant soit peu informé de la vie intellectuelle montréalaise des années 2000 n'aura aucun mal à reconnaître sous ce nom d'emprunt le défunt Lothar Baier (1942-2004) ${ }^{17}$. Non

\footnotetext{
16 À un autre moment du récit, alors qu'elle ne veut toujours pas donner son nom à un revendeur de drogue, elle prendra celui de Monica, une activiste liée au mouvement opéraïste italien (cf. infra).

17 Quelques éléments de la biographie de Lothar Baier se découvriront au fil de mon analyse. Mentionnons seulement pour le moment qu'il s'agit d'un journaliste et d'un écrivain qui a joué un rôle important sur la scène intellectuelle allemande, participant à des entreprises éditoriales liées à la gauche contestataire. Baier était aussi un fin analyste des relations culturelles et politiques franco-allemandes. À la fin de sa carrière, il avait émigré à Montréal, où il s'était taillé une place enviable au sein de l'intelligentsia québécoise. Pour le lecteur allemand, le nom de Lukas Bauer pourrait également faire écho
} 
seulement le nom romanesque est transparent, mais les trajectoires des personnages sont comparables. Ici aussi, toutefois, des techniques de floutage viennent déjouer toute tentative de rabattre exactement le personnage sur la personne. Par exemple, Baier est mort à 62 ans alors que Bauer, dans le roman, a vécu jusqu'à 68 ans ${ }^{18}$; Baier a été retrouvé sans vie dans son appartement alors que Bauer, selon la narratrice, se serait noyé ( $F C: 55)$; l'écrivain réel a écrit directement en français un essai autobiographique intitulé Un Allemand né de la dernière guerre (1989 [1986]) et, en allemand, un roman, également autobiographique, ayant pour titre Le délai (1992 [Jahresfrist, 1985]), tandis que l'écrivain fictif est l'auteur d'une monumentale autobiographie publiée sous le titre Les liens.

En la personne de Lukas Bauer, le réel fait donc irruption dans la fiction sous une forme déguisée. Plusieurs informations biographiques concernant Baier, surtout puisées dans son essai et dans son roman autobiographiques, se mêlent en effet à des inventions qui représentent tantôt des extrapolations à partir de ce qui est connu de la vie du modèle, tantôt de franches déviations. Le portrait de l'enfance et de l'adolescence très libres de Bauer (FC:51-52), soustraites à la surveillance et à la surprotection des adultes, est ainsi tout droit tiré de celui qu'en fait Baier au premier chapitre d'Un Allemand né de la dernière guerre; l'origine ouvrière, en revanche, est fausse, Baier étant plutôt issu d'une famille d'enseignants. Le roman de Frédérick rend compte avec exactitude de la révolte de Baier contre les pères nazis ou complices du nazisme, ou encore contre la normalisation accélérée d'une République fédérale peu regardante sur le passé de ses hauts dirigeants (FC:65-66); mais il s'agit là, au vrai, d'un trait générationnel

à un roman pour la jeunesse de Willi Fährmann très populaire, Der lange Weg des Lukas B. Il y est question de l'émigration d'un jeune Allemand en Amérique - on pourrait donc y voir aussi un parallèle avec Baier.

${ }^{18}$ La narratrice le fait naître en 1938 dans le roman, ce qui le ferait mourir, s'il avait vécu 68 ans, en 2006 plutôt qu'en 2004. 
plus que d'un caractère spécifique à Baier-Bauer. La narratrice s'attache enfin au rapport particulier de Bauer à la France, qui apparaît clairement dans les ouvrages du Baier réel, notamment dans Un Allemand né de la dernière guerre (sous-titré Essai à l'usage des Français), Le délai, récit de l'établissement d'un Allemand dans un coin reculé de l'Ardèche, et L'entreprise France (1989 [Firma Frankreich. Eine Betriebsbesichtigung, 1988]).

Les développements sur les liens de Bauer avec l'extrême-gauche allemande renvoient à un aspect capital de la vie de Baier. Ce dernier a été un observateur attentif et souvent critique de la radicalisation de la gauche allemande issue du mouvement étudiant qui allait conduire, entre autres, à l'émergence de la Rote Armee Fraktion (RAF). Le roman montre bien la position d'outsider qui a été celle de Bauer-Baier, non sans extrapoler sur de présumées relations amoureuses avec des femmes terroristes qui lui auraient reproché la tiédeur de ses convictions révolutionnaires. La vision, ici, est nettement romantique, et sans doute plus proche de la perception de la jeune narratrice que de celle d'un Bauer-Baier dépeint comme étant revenu de la plupart de ses illusions. Bauer aurait ainsi été amoureux de Nina Knodas, arrêtée en 1970 pour sa participation à l'attaque des trois banques de Berlin (FC: 66), et d'Ilaria Libardi, membre du groupe armé italien Prima Linea (FC : 75). Ces noms inventés s'inscrivent dans un système mixte, pour ainsi dire, puisque, pour évoquer les « années de plomb » en Allemagne et en Italie, le texte a également recours à des noms et à des personnages véritables tels que Benno Ohnesorg ${ }^{19}$ et Pietro Valpreda ${ }^{20}$. L'histoire se tisse ainsi à la fiction sans trop d'accrocs, par l'insertion soit de personnages réels très secondaires, soit d'acteurs historiques de premier plan transposés en figures en partie fictionnalisées.

\footnotetext{
${ }_{19}$ Étudiant tué par un policier ouest-allemand (dont on apprit plus tard qu'il travaillait pour la Stasi) lors d'une manifestation d'étudiants à Berlin-Ouest, en 1967, contre le shah d'Iran; cet événement est considéré comme le déclencheur de la radicalisation de la gauche en Allemagne.

${ }^{20}$ Anarchiste italien faussement accusé d'être l'auteur de l'attentat de la piazza Fontana à Milan en 1969.
} 
Au-delà de la composante factuelle des rapports entre le Bauer de fiction et le Baier réel, de nombreux parallèles plus généraux peuvent être établis entre le personnage romanesque et la personne avérée. Selon le témoignage de Frédérick elle-même, celle-ci n'aurait qu' « entrevu [Baier] à Québec», mais elle aurait lu tous ses livres pour préparer son premier roman, La poupée de Kokoschka (Frédérick et Massoutre 2014: n. p.), ce qui est à la fois plus et moins que la relation évoquée dans le roman. Plus, car la narratrice de Forêt contraire n'a lu aucun livre de Bauer au début du récit et n'aura lu, à la fin, que son autobiographie; et moins, car, dans la fiction, elle a fait bien davantage qu'«entrevoir» l'écrivain, elle l'a rencontré à quelques reprises, a décliné quelques-unes de ses invitations, l'a côtoyé suffisamment pour deviner sa détresse et son mal de vivre. De fait, le portrait que dresse "Sophie » de l'écrivain hessois au bout de son rouleau apparaît exact : le sourire et l'air espiègle de plus en plus entamés par le poids d'une étrangeté au temps présent correspondent tout à fait à l'image de Baier qu'en donnent, dans les notices nécrologiques, ceux qui l'ont bien connu. Cette faille que la narratrice décèle d'emblée en Bauer, son «gouffre profond» (FC: 77$)$ où elle craint elle-même de sombrer sont précisément ce qui la retient de nouer une relation plus étroite avec lui et ce qui, des années après, l'incite à repousser la lecture des Liens.

Enfin, le rapport à Baier se déploie sur le plan des résonances et des similitudes entre Le délai, son roman autobiographique, et Forêt contraire. Entre l'Ardèche profonde où va s'enterrer un intellectuel allemand en rupture de ban ${ }^{21}$ et la forêt québécoise des Bois-Francs autour d'Inverness, il y a bien sûr des différences notables, même si l'on peut envisager ces deux régions comme des territoires également contraires, comme des lieux de contraintes et de contrariétés, où se produisent donc des choses contraires aux attentes, où les quêtes dévient.

${ }^{21}$ Baier avait effectivement tenté - sans succès - de s'installer dans l'Ardèche. 
Ainsi l'Allemand en année sabbatique comme la narratrice échappée de Paris se construisent-ils de nouvelles chaînes en se reliant étroitement à des «maison[s] contraire[s]»(FC: 13), pour reprendre l'expression de la narratrice, et en s'insérant dans un nouveau tissu social, fût-il passablement élimé, alors qu'ils cherchaient avant tout à s'isoler, à rompre. Les ressemblances ne s'arrêtent pas là : les deux romans mettent en scène des personnages solitaires qui se sont réfugiés dans des abris qui faillissent à les protéger - fenêtres rongées, toiture écroulée de la vieille ferme dans Le délai (1992 [1985] : 20)22, chalet instable et pourri dans Forêt contraire -, où les souvenirs insistants menacent le désir d'amnésie et de recommencement, où le délabrement général apparaît, pour dire comme le narrateur du Délai, à la fois comme une menace et un défi $(D: 26)$. C'est à travers le motif de la maison, bien plus que par de très vagues similitudes entre certaines dispositions personnelles, que les protagonistes se font surtout écho. Quand le voisin André propose à «Sophie » de quitter le vieux chalet menaçant ruine et de lui construire un nouveau refuge, tout de suite elle pense à « un abri pour le livre de Lukas » (FC: 74). «Et on se réinventera, Lukas Bauer et moi, en elle » (FC:75), continue-t-elle, la maison devenant le lieu d'une rencontre enfin possible ( $F C: 99$ ), d'une sorte de réparation (la narratrice n'ayant pas même tenté de «sauver» Bauer). Le délai accorde aussi une grande importance à l'habiter : la trame du roman consiste, pour l'essentiel, dans le récit étape par étape de la restauration d'une maison qui, sous ses dehors rustiques, se révèle en fait fragile, ouverte aux quatre vents, à l'opposé de la forteresse $(D: 63,64)$ que le personnage espérait y trouver.

Encore plus frappant est le dénominateur commun que constitue l'apparition, dans les deux romans, de personnes réelles présentes-absentes, le

\footnotetext{
${ }^{22}$ Dorénavant, les références à cet ouvrage seront notées par le sigle $D$ et le folio, entre parenthèses dans le corps du texte.
} 
rôle de Bauer-Baier dans Forêt contraire étant joué dans Le délai par Paul Nizan ${ }^{23}$. Dans le livre de Baier, Nizan commence par se manifester discrètement, par une citation non attribuée $(D: 17)$ puis par une citation dûment renvoyée à son auteur $(D: 28)$. «Paul Nizan » devient vite «Paul» tout court $(D: 29)$, une figure ainsi détachée de son nom de gloire pour se muer en compagnon intime qui va guider le personnage dans son appréhension de sa situation présente comme dans celle de son passé. Plus tard, alors que le narrateur quitte sa maison ardéchoise pour se rendre aux funérailles de son frère en Allemagne et fait étape dans une bourgade dénommée "Laville », survient un événement de nature fantastique : devant un arrêt de car, il aperçoit Nizan, qui ne serait pas mort à la guerre à l'âge de 35 ans mais qui serait là, silhouette bedonnante, casquette d'ouvrier, "yeux qui louchaient légèrement derrière ses verres de lunettes » (D: 131). Peu après, parcourant un livre sur la Résistance où il est question d'un bombardier allié qui se serait écrasé sur un rocher au-dessus de Laville, le narrateur échafaude l'hypothèse hasardeuse selon laquelle Nizan aurait été un passager de l'avion qui aurait survécu à l'écrasement. Ayant échappé une seconde fois à la mort après Recques-sur-Hem où le vrai Nizan a été tué, l'écrivain aurait décidé de rester sur place et de ne plus se mêler de rien, ce qui expliquerait donc sa présence dans la petite ville $(D: 136-138)$.

À son retour d'Allemagne, le narrateur regagne Laville à la première occasion pour tenter de surprendre la réapparition de l'auteur d'Aden Arabie. Il n'est pas long à retracer un Nizan de plus de 70 ans, faux évidemment, toujours vivant plus de 40 ans après sa mort « officielle », avec qui il va régler ses comptes dans un chapitre écrit comme une longue adresse à un interlocuteur muet ${ }^{24}$. Dans ce très beau texte, le narrateur revient sur l'expérience existentielle de Nizan, sur sa jeunesse studieuse, son engagement communiste et sa rupture avec le parti à la

\footnotetext{
${ }^{23}$ Notons que Baier a traduit Nizan en allemand.

${ }^{24}$ Le vingt et unième chapitre $(D: 177-186)$ sur un total de vingt-quatre.
} 
signature du pacte germano-soviétique, sur son refus de s'expliquer et son départ au front où il meurt dès 1939, figé pour toujours dans sa légende de clairvoyant et de renégat du communisme. Sur le ton de l'hommage et du reproche, le narrateur lui reconnaît l'intelligence d'avoir su «survivre à l'erreur » de l'adhésion au communisme $(D: 178)$ tout en l'accusant de s'être « un peu facilité la tâche $[\ldots]$ en [ $\mathrm{s}^{\prime}$ ] esquivant sans mot dire » $(D: 178)$. Surtout, il voit en Nizan un père spirituel ayant suppléé aux pères réels laminés par le nazisme et par la guerre - un père qui semble avoir raison pour l'éternité et qui ne peut, de ce fait, être liquidé. On arrive d'autant moins à se débarrasser de ce père encombrant et héroïque que les utopies révolutionnaires ont vécu, qu' «il n'y a plus d'Espagne pour laquelle il vaille la peine de se battre » $(D: 184)$, qu'il ne reste que la vie individuelle à réformer, et par des moyens en comparaison dérisoires, qu'il s'agisse du retour à la campagne, du refus du capital ou du repli sur l'intimité.

Cette fictionnalisation de la personne réelle trouve un pendant spectaculaire dans Forêt contraire. En effet, à la fin du roman, André propose à «Sophie » de « rencontrer Lukas Bauer» $(F C: 130)$ par le truchement de masques neutres qui leur permettront de « jouer avec le temps et les souvenirs » (FC:130). André semble tout désigné pour être le meneur d'un semblable jeu, lui qui, remarque la narratrice, «se meut dans la réalité avec l'aisance de la fiction » (FC: 71), avec la souplesse du comédien capable d'accueillir des personnages inventés. Il y aura deux séances où les personnages deviendront des "pantins de la fiction », des «marionnette[s] » (FC: 142). «Sophie », par des comportements provocateurs qui dérogent aux règles du jeu, va ainsi tenter de faire advenir, de forcer une rencontre avec Bauer qui, dans la vie, n'a pas vraiment eu lieu; mais cette rencontre ne pourra se produire qu'avec le Bauer imaginé par André, qui lui ne l'a pas connu. Et elle se terminera par ce qui ressemble à un viol, sans qu'on puisse bien savoir si c'est Bauer ou André qui le perpètre. Après ce dénouement, la narratrice écrira un message de «rupture » pour Lukas qu'elle ira déposer 
dans la boîte aux lettres d'André, désignant de la sorte une fiction à double fond, sinon à double entente. Puis elle mettra le feu à la cabane offerte par André avant de quitter définitivement Inverness.

Cette ultime péripétie apparaît, en définitive, tout à fait logique du point de vue romanesque car, au terme du récit, Lukas Bauer n'a plus besoin d'un abri «physique »: c'est le roman de Frédérick qui, par le recours à une clé transparente et à des effets de miroirs nombreux avec l'œuvre de Baier, est devenu en quelque sorte son reliquaire...

\section{Conclusion}

Les fictions dont il a été question ici, si elles constituent sans doute, par certains côtés, des romans à clés, invitent à davantage qu'à une simple opération de décryptage des noms cachés. La transparence de la plupart des noms, chez Hamelin comme chez Frédérick, semble d'ailleurs indiquer que l'encryptage n'était pas un enjeu fondamental de l'élaboration romanesque. Pourquoi dès lors avoir eu recours à ce "genre » décrié pour, dans un cas, relire l'histoire récente du Québec et, dans l'autre, sonder les possibles d'une «non-rencontre » avec un intellectuel qui, après coup, semble avoir hanté l'auteure?

Il y a fort à parier que c'est justement en raison des passages qu'il instaure entre le réel et la fiction qu'Hamelin a choisi la forme du roman à clés. Renommer, parfois de manière loufoque, des personnages historiques, c'est non seulement les convoquer dans la fiction, c'est aussi leur conférer une dimension romanesque qui remet en jeu le pur niveau des faits, les doter d'une aura fictive comme si leur place et leur rôle dans l'histoire restaient sujets à caution, légèrement indéterminés et par conséquent disponibles à tous les réinvestissements herméneutiques. Plus largement, l'usage des clés invite à revoir les rapports entre le réel et la fiction, à remettre en cause les frontières censées les 
cloisonner. La chose apparaît on ne peut plus justifiée dans un récit qui entend déboulonner les fictions d'Octobre 1970 élaborées par les uns et les autres en produisant une fiction de rechange, une fiction plus réaliste de se savoir en partie fictive. Chez Hamelin, le changement des noms semble ainsi opérer sur deux plans : il sape aussi bien le fondement réel sur lequel reposent les récits de la crise d'Octobre que le réalisme littéraire, mis à mal entre autres par l'auto-ironie et le grotesque.

Le contexte plus immédiatement fictionnel et pour l'essentiel intimiste de Forêt contraire confère une dimension tout à fait différente à l'irruption de la personne réelle. Le personnage de Bauer-Baier apporte une assise autobiographique à la narration - l'auteure a connu Baier de loin - qui permet au roman de se déployer d'abord comme une quête de l'autre, puis comme une entreprise de réparation. Sur un autre plan, toutefois, l'établissement de parallèles et de symétries entre le mal de vivre de la narratrice et celui de Bauer, entre le roman autobiographique de ce dernier et celui qu'elle est en train de rédiger, entre l'usage de la figure réelle de Nizan et celui de la figure réelle de Bauer-Baier, rend compte d'une captation beaucoup plus profonde d'une personne et d'une œuvre qu'on pourrait qualifier de « sources ». Forêt contraire est certes un hommage à Baier, mais elle est davantage encore une excroissance, une expansion, bien qu'elle ne se réduise pas à un parasitage de la réalité autobiographique d'autrui. En effet, elle la retravaille, elle la décale, si bien qu'inventer Bauer, c'est certes convoquer Baier, mais plus encore le reconfigurer pour les besoins de la cause romanesque: dans le roman de Frédérick, il est nécessaire que Bauer ait aimé des femmes terroristes, il faut qu'il ait été fils d'ouvrier, il importe que Baier perde son véritable nom, car il faut qu'il soit un miroir de la révolte, de l'amnésie, de l'abandon amoureux et charnel qui animent la narratrice. 
Écrire la personne réelle, en toute exactitude ou en la fabulant, en la désignant par son véritable anthroponyme ou en la déguisant légèrement, ce n'est donc pas ici seulement donner au roman le poids du réel, mais produire le réel sur la scène de la fiction : une scène qui, pour ces écrivains, semble englober le monde.

\section{Bibliographie}

Baier, L. (1992 [1985]). Le délai. Arles, Actes Sud.

Baier, L. (1989 [1988]). L'entreprise France. Paris, Calmann-Lévy.

Baier, L. (1989 [1986]). Un Allemand né de la dernière guerre. Essai à l'usage des Français. Paris, Calmann-Lévy.

Caron, L. (1990). Les fils de la liberté, t. 3. Le coup de poing. Montréal, Boréal.

Dion, R. (2018). Des fictions sans fiction ou le partage du réel. Montréal, Presses de l'Université de Montréal.

Dion, R. (2015a). «Le passé historique dans les écritures québécoises du présent (Hamelin, Ouellette-Michalska, Leclerc, Mavrikakis) ». Letras de Hoje (Porto Alegre), vol. 50, no 2 (avril-mai), p. 168-178.

Dion, R. (2015b). « Présences d'Octobre. Variations littéraires autour d'un épisode de l'histoire québécoise (Louis Hamelin, Carl Leblanc)». Voix et Images, no 121 (automne), p. 87-100.

Ferretti, A. (1990). "Octobre de lumière ». Dans La vie partisane. Montréal, l'Hexagone, p. 47-66.

Ferron, J. (1969). Le ciel de Québec. Montréal, Éditions du Jour. 
Frédérick, H. (2015 [2014]). Forêt contraire. Montréal, Héliotrope (coll. «Série "P"»).

Frédérick, H. (2014 [2010]). La poupée de Kokoschka. Montréal, Héliotrope.

Frédérick, H. et G. Massoutre. (2014). « Les créatures libres d’Hélène Frédérick. Rencontre avec une romancière audacieuse, qui invente Munich aussi bien qu'Inverness » [entrevue]. Le Devoir, 22 mars.

Glinoer, A. et M. Lacroix. (2014). Romans à clés. Les ambivalences du réel. Liège, Presses universitaires de Liège.

Hamelin, L. (2014). Fabrications. Essai sur la fiction et l'histoire. Montréal, Presses de l'Université de Montréal.

Hamelin, L. (2012 [2010]). La constellation du Lynx. Montréal, Boréal (coll. «Boréal Compact»).

Hamelin, L. et N. Mercier (2010). «La bombe Hamelin ». L'Actualité, 29 septembre.

Lacroix, M. (2014). «Imaginaire, légendaire, fictif : romans à clés et fiction de la vie littéraire ». Dans A. Glinoer et M. Lacroix (dir.), Romans à clés. Les ambivalences $d u$ réel. Liège, Presses universitaires de Liège, p. 11-20.

Latham, S. (2014 [2009]). "Réalité, fiction et plaisir. Introduction du livre The Art of Scandal. Modernism, Libel Law and the Roman à Clef». Dans A. Glinoer et M. Lacroix (dir.), Romans à clés. Les ambivalences du réel. Liège, Presses universitaires de Liège, p. 21-42.

Nardout-Lafarge, É. (2013). «La mémoire de Ferron dans La constellation du Lynx de Louis Hamelin ». Dans I. Daunais (dir.), La mémoire du roman. Montréal, Presses de l'Université de Montréal, p. 177-190.

Pelletier, J. (2007 [2002]). «Octobre 1970 : la leçon de la fiction ». Dans Question nationale et lutte sociale, la nouvelle fracture. Écrits à contre-courant 2. Québec, Nota bene, p. 171-182. 
Pelletier, J. (1995 [1982]). «La crise d'Octobre 1970 et la littérature québécoise ». Dans Le poids de l'histoire. Littérature, idéologies, société du Québec moderne. Québec, Nuit blanche éditeur, p. 141-162.

Shields, D. (2010). Reality Hunger. A Manifesto. New York, Vintage Books.

Turgeon, P. (1990). Un dernier blues pour Octobre. Montréal, Libre Expression.

Wagner, F. (2008). «Perturbations onomastiques: l'onomastique romanesque contre la mimèsis». Dans Y. Baudelle (dir.), Onomastique romanesque, Narratologie, no 9. Paris, L'Harmattan, p. 17-42. 